\title{
COMBINATORIAL NUMBERS IN BINARY RECURRENCES
}

\author{
T. KOVÁCS
}

\begin{abstract}
We give several effective and explicit results concerning the values of some polynomials in binary recurrence sequences. First we provide an effective finiteness theorem for certain combinatorial numbers (binomial coefficients, products of consecutive integers, power sums, alternating power sums) in binary recurrence sequences, under some assumptions. We also give an efficient algorithm (based on genus 1 curves) for determining the values of certain degree 4 polynomials in such sequences. Finally, partly by the help of this algorithm we completely determine all combinatorial numbers of the above type for the small values of the parameter involved in the Fibonacci, Lucas, Pell and associated Pell sequences.
\end{abstract}

\section{INTRODUCTION}

There are many papers about values of a polynomial $p(x) \in \mathbb{Q}[x]$ (taken at integer values of $x$ ) in a binary linear recurrence sequence $U$. The first such results dealt with the case where $U$ is a special sequence and $p(x)=x^{m}$ with some $m \geq 2$. That is, we are interested in terms of $U$ which are perfect powers. In 1962 Ogilvy [22], one year later Moser and Carlitz [20], and Rollett [30] proposed the following problem: determine all squares in the Fibonacci sequence $F$. The problem was solved by Cohn [7, 8] and Wyler [39] who independently proved with elementary methods that the only squares in the Fibonacci sequence are $F_{0}=0, F_{1}=F_{2}=1, F_{12}=144$. Later, Alfred [1] and Cohn [9] determined the squares in the Lucas sequence $L$. Pethő [25] and Cohn [10] independently determined the perfect powers in the Pell sequence. Recently, Bugeaud, Mignotte and Siksek [6] showed that the perfect powers in the Fibonacci and Lucas sequences are exactly $F_{0}=$ $0, F_{1}=F_{2}=1, F_{6}=8, F_{12}=144$, and $L_{1}=1, L_{3}=4$, respectively.

2000 Mathematics Subject Classification. Primary 11B37, secondary 11B83, $11 \mathrm{Y} 50$.

Key words and phrases. Binary recurrence sequences, polynomial values, combinatorial numbers. 
Another branch of problems is about triangular numbers in recurrence sequences, i.e. we take the polynomial $p(x)=\frac{x(x+1)}{2}$. Hoggatt stated the conjecture that there are only five triangular Fibonacci numbers. In 1989 Ming [18] proved that this conjecture is true. Furthermore, Ming [19] and McDaniel [17] determined the triangular numbers in the Lucas and Pell sequences, respectively. In [34] Szalay described all values of the polynomials $S_{2}(x)$ and $S_{3}(x)$ in the Fibonacci, Lucas and Pell sequences, where $S_{k}(x)$ denotes the sum of $k$ th powers up to $x-1$. Further, he listed all numbers of the form $\left(\begin{array}{l}x \\ 4\end{array}\right)$ in the Fibonacci and Lucas sequences, as well. As a generalization of the previous results, Tengely [37] recently determined the $g$-gonal numbers in the Fibonacci, Lucas, Pell and associated Pell sequences for $g \leq 20$.

The above mentioned results give complete solutions of the problem in case of certain sequences $U$ and polynomials $p$. Beside them there are several results in the literature which provide effective upper bounds for the solutions under certain assumptions. The most extensively investigated case is about perfect powers, i.e. where $p(x)=x^{m}$ with some $m \geq 2$. Instead of trying to survey the extremely huge literature we only refer to the book [31] and the references given there. Finally, we mention that Szalay [34] provided an algorithm for the complete description of the values of a polynomial $p(x)$ of degree 3 in a binary recurrence sequence $U$ under some assumptions.

In this paper we prove three theorems concerning the values of some polynomials in binary recurrence sequences. First we provide an effective finiteness theorem for certain combinatorial numbers, namely for binomial coefficients, products of consecutive integers, power sums and alternating power sums in binary recurrence sequences, under some assumptions. The proof of this theorem is based on Baker's method together with certain results of Brindza [4], Ping-Zhi [26], Pintér and Rakaczki [27] and Rakaczki [29]. Our second result is an extension of the above mentioned result of Szalay. More precisely, it provides an efficient algorithm for determining the values of certain degree 4 polynomials in binary recurrence sequences, under some assumptions. In particular, we implemented the main part of our algorithm in Magma [3]. Our program can be downloaded from the homepage www.math.klte.hu/ tkovacs or the author can send it if requested. Finally, partly by the help of this algorithm we give all combinatorial numbers mentioned above for the small values of the parameter involved in the Fibonacci, Lucas, Pell and associated Pell sequences. We mention that to prove the latter result we reduce the problem to elliptic and more generally to genus 1 equations. There is an efficient method to 
determine the solutions of such equations developed by Gebel, Pethő and Zimmer [12] and independently by Stroeker and Tzanakis [32]. Later, the method was further improved and generalized by Stroeker and Tzanakis (see [33] and the references given there). Moreover, the program package Magma contains procedures (based on these results) to resolve such equations. Hence we can ultimately use Magma to solve our particular equations. For other combinatorial problems solved in a similar way, we refer to [13] and [16] and the references given there.

\section{Notation}

Let $U=\left\{U_{n}\right\}_{n=0}^{\infty}$ be a binary recurrence sequence defined by the initial terms $U_{0}, U_{1} \in \mathbb{Z}$ and the recurrence relation

$$
U_{n}=A U_{n-1}+B U_{n-2} \quad(n \geq 2)
$$

where $A, B$ are non-zero integers. Let $\alpha$ and $\beta$ denote the zeros of the companion polynomial $x^{2}-A x-B$ of $U$. Further, let $D=A^{2}+4 B$ be the discriminant of $U$ and

$$
a_{u}=U_{1}-\beta U_{0}, \quad b_{u}=U_{1}-\alpha U_{0}, \quad C=a_{u} b_{u}=U_{1}^{2}-A U_{0} U_{1}-B U_{0}^{2} .
$$

The sequence $U$ is called non-degenerate if $C \neq 0$ and $\alpha / \beta$ is not a root of unity. It is well-known that if $U$ is non-degenerate then for all $n=0,1, \ldots$ we have

$$
U_{n}=\frac{a_{u} \alpha^{n}-b_{u} \beta^{n}}{\alpha-\beta} .
$$

From this point on we assume that $B= \pm 1$ and that $U$ is nondegenerate. Then as it is also well-known, $U$ has a so-called associate sequence $V=\left\{V_{n}\right\}_{n=0}^{\infty}$ for which

$$
V_{n}^{2}-D U_{n}^{2}=4 C(-B)^{n}
$$

holds for all $n=0,1, \ldots$ Observe that by our assumption $B= \pm 1$, we have $(-B)^{n}= \pm 1$. Further, note that $V_{0}=2 U_{1}-A U_{0}, V_{1}=$ $A U_{1}+2 B U_{0}$ and $V$ satisfies the same recurrence relation as $U$.

Beside dealing with general sequences $U$ we consider combinatorial numbers in certain special famous sequences, too. Let $F, L, P$ and $Q$ denote the Fibonacci, Lucas, Pell and associated Pell sequence, respectively. These sequences are defined by

$$
\begin{array}{llll}
F_{0}=0, & F_{1}=1, & F_{n}=F_{n-1}+F_{n-2} & (n \geq 2), \\
L_{0}=2, & L_{1}=1, & L_{n}=L_{n-1}+L_{n-2} & (n \geq 2), \\
P_{0}=0, & P_{1}=1, & P_{n}=2 P_{n-1}+P_{n-2} & (n \geq 2), \\
Q_{0}=1, & Q_{1}=1, & Q_{n}=2 Q_{n-1}+Q_{n-2} & (n \geq 2) .
\end{array}
$$

Now we give what kind of combinatorial numbers we are interested in. Beside binomial coefficients, we consider power sums, alternating 
power sums and products of consecutive integers as well. We use the following (standard) notation. For all $k, x \in \mathbb{N}$ let

$$
\begin{gathered}
S_{k}(x)=1^{k}+2^{k}+\ldots+(x-1)^{k}, \\
T_{k}(x)=-1^{k}+2^{k}-\ldots+(-1)^{x-1}(x-1)^{k}, \\
\Pi_{k}(x)=x(x+1) \cdots(x+k-1) .
\end{gathered}
$$

We mention that $S_{k}(x)$ is a polynomial of degree $k+1$, while $T_{k}(x)$ and $\Pi_{k}(x)$ are polynomials of degree $k$.

\section{NEW RESUlts}

We use the previous notation. Further, recall that $B= \pm 1$ and $U=\left\{U_{n}\right\}_{n=0}^{\infty}$ is non-degenerate. All our results concern the equation

$$
U_{n}=p(x)
$$

in integers $n, x$ with $n \geq 0$. For the sake of completeness we also take care of the solutions with $x \leq 0$, although these solutions usually do not have combinatorial meanings.

First we give an effective result for the solutions of (2) which is valid for general $U$.

Theorem 3.1. Let $k \geq 2$ and $p(x)$ be one of the polynomials $S_{k-1}(x)$, $T_{k}(x), \Pi_{k}(x),\left(\begin{array}{l}x \\ k\end{array}\right)$. If either $k=2$ or $p(x)$ is one of $S_{2}(x), \Pi_{3}(x),\left(\begin{array}{l}x \\ 3\end{array}\right)$, then further assume that $B=1$. Then the solutions $n, x$ of equation (2) satisfy $\max (n,|x|)<c_{0}(U, k)$, where $c_{0}(U, k)$ is an effectively computable constant depending only on $U$ and $k$.

Obviously, the assumption $k \geq 2$ cannot be omitted. The next proposition shows that the condition $B=1$ in the special cases of the theorem is necessary as well.

Proposition 3.1. Let $U$ be the sequence defined by $B=-1$ and by the values $U_{0}, U_{1}, A$ given in the $i$ th row of Table 1 , for any $i \in\{1,2,3,4,5\}$. Further, let $p(x)$ be a polynomial from the last column of the ith row of Table 1. Then equation (2) has infinitely many solutions.

Remark. If (2) has infinitely many solutions then the set of solutions has some special structure. This structure has been described by Nemes and Pethő [21], see Theorem 3 (cf. also [23], [24]). It turns out that the solutions $x$ belong to certain recurrence sequences, while the solutions $n$ come from some arithmetic progressions. For details see [21], [23] and [24]. Furthermore, to find the examples provided by Table 1, the above mentioned Theorem 3 of [21] can also be used. Since the assumptions of this theorem are not sufficient (see Remark 2 of [21]), it remains necessary to show that in these cases (2) really has infinitely many 


\begin{tabular}{|c|c|c|c|}
\hline$U_{0}$ & $U_{1}$ & $A$ & $p(x)$ \\
\hline \hline 1 & 253 & 254 & $S_{1}(x), T_{2}(x),\left(\begin{array}{c}x \\
2\end{array}\right)$ \\
\hline 2 & 506 & 254 & $\Pi_{2}(x)$ \\
\hline 1 & 3759787041401 & 3760028828350 & $S_{2}(x)$ \\
\hline 7770 & 455962704852690 & 58682458798 & $\left(\begin{array}{l}x \\
3\end{array}\right)$ \\
\hline 46620 & 2735776229116140 & 58682458798 & $\Pi_{3}(x)$ \\
\hline
\end{tabular}

TABLE 1

solutions. We also mention that for all polynomials that occur in Table 1 using the method in [21] many more binary recurrence sequences $U$ can be constructed such that equation (2) has infinitely many solutions.

As we mentioned above, Szalay [34] gave an algorithm for the resolution of $(2)$ in the case when $p(x)$ is a polynomial of degree 3 . We extend this result to the degree 4 case. For this purpose we need some further notation. Let $p(x) \in \mathbb{Q}[x]$ be a polynomial of degree 4 and write

$$
p(x)=A_{0} x^{4}+A_{1} x^{3}+A_{2} x^{2}+A_{3} x+A_{4} .
$$

Suppose that the coefficients of $p$ fulfill the relations

$A_{0}=\frac{a}{e}, A_{1}=\frac{4 a b}{e}, A_{2}=\frac{6 a b^{2}+c}{e}, A_{3}=\frac{4 a b^{3}+2 b c}{e}, A_{4}=\frac{a b^{4}+b^{2} c+d}{e}$,

with some integers $a, b, c, d, e, a e \neq 0$. Then we have

$$
p(x)=\frac{a(x+b)^{4}+c(x+b)^{2}+d}{e} .
$$

Write $x_{1}=x+b$ and let $y=V_{n}$ where $V=\left\{V_{n}\right\}_{n=0}^{\infty}$ is the associate sequence of $U$. Then by (1) we get

$$
y^{2}-D\left(\frac{a x_{1}^{4}+c x_{1}^{2}+d}{e}\right)^{2}=4 C(-B)^{n},
$$

which yields

$$
Y^{2}=h_{4} X^{4}+h_{3} X^{3}+h_{2} X^{2}+h_{1} X+h_{0},
$$

where

$$
\begin{gathered}
Y=e y, \quad X=x_{1}^{2}, \quad h_{4}=a^{2} D, \quad h_{3}=2 a c D \\
h_{2}=\left(c^{2}+2 a d\right) D, \quad h_{1}=2 c d D, \quad h_{0}=d^{2} D+4 e^{2} C(-B)^{n} .
\end{gathered}
$$


Equation (3) in general is of genus 1, therefore the so-called Ellog method of Stroeker and Tzanakis [33] can be used to determine all its integral solutions. In particular, using the program package Magma, equation (3) can be solved completely in concrete cases. If $h_{0}$ is a perfect square then (3) can be solved directly by the procedure IntegralQuarticPoints. Putting together some tools and results about genus 1 curves, we give an efficient method for the resolution of (3) in the general case. For the description of the method see the proof of Theorem 3.2. (Further, as we mentioned we implemented our algorithm in Magma, too.) From the solutions, the values $x$ and the indices $n$ can be easily determined.

Summarizing the above argument, we get

Theorem 3.2. Using the previous notation, suppose that $8 a D d(2 a d-$ $\left.c^{2}\right) \neq-64 a^{2} C \pm e^{2}-c^{4} D$. Then equation (2) has only finitely many solutions $n, x$ and these solutions can be effectively determined.

Our final result completely describes the above type combinatorial numbers for the small values of the parameter $k$ in some well-known binary recurrence sequences.

Theorem 3.3. Let $U \in\{F, L, P, Q\}$ and $p(x) \in\left\{S_{1}(x), S_{2}(x), S_{3}(x)\right.$, $\left.T_{2}(x), T_{4}(x), \Pi_{2}(x), \Pi_{3}(x), \Pi_{4}(x),\left(\begin{array}{l}x \\ 2\end{array}\right),\left(\begin{array}{l}x \\ 3\end{array}\right),\left(\begin{array}{l}x \\ 4\end{array}\right)\right\}$. Then the solutions $n, x$ of equation (2) are exactly those which are contained in Table 2. The sign "-" shows that the actual equation has no solution. Further, the references given in the table indicate that the corresponding equation was solved in the appropriate paper.

Remark. The complete solution of the equation $U_{n}=T_{3}(x)$ remains open. In this case by relation (1) and with the substitution $y=V_{n}$ we get the equation

$$
y^{2}=D \frac{4 x^{6}-12 x^{5}+9 x^{4}}{16}+4 C(-B)^{n}
$$

if $x$ is even and

$$
y^{2}=D \frac{4 x^{6}-12 x^{5}+9 x^{4}+4 x^{3}-6 x^{2}+1}{16}+4 C(-B)^{n}
$$

if $x$ is odd. These equations are of genus 2 thus neither Szalay's method nor our algorithm given in the proof of Theorem 3.2 can be used to solve them. 


\begin{tabular}{|l|l|l|l|l|}
\hline$=$ & $F_{n}$ & $L_{n}$ & $P_{n}$ & $Q_{n}$ \\
\hline$S_{1}(x)$ & {$[18]$} & {$[19]$} & {$[17]$} & $\begin{array}{l}(0,-1),(0,2),(1,-1), \\
(1,2),(2,-2),(2,3)\end{array}$ \\
\hline$S_{2}(x)$ & {$[34]$} & {$[34]$} & {$[34]$} & $(0,2),(1,2)$ \\
\hline$S_{3}(x)$ & {$[34]$} & {$[34]$} & {$[34]$} & $\begin{array}{l}(0,-1),(0,2), \\
(1,-1),(1,2)\end{array}$ \\
\hline$T_{2}(x)$ & $\begin{array}{l}(0,0),(0,1),(1,-1), \\
(2,-1),(4,3), \\
(8,7),(10,11)\end{array}$ & $\begin{array}{l}(1,-1),(2,3), \\
(18,-107)\end{array}$ & $(1,-1)$ & $(0,-1),(1,-1),(2,3)$ \\
\hline$T_{4}(x)$ & $\begin{array}{l}(0,0),(0,1), \\
(1,-1),(2,-1)\end{array}$ & $(1,-1)$ & $(0,0),(0,1),(1,-1)$ & $(0,-1),(1,-1)$ \\
\hline$\Pi_{2}(x)$ & $\begin{array}{l}(0,-1),(0,0), \\
(3,-2),(3,1)\end{array}$ & - & $\begin{array}{l}(0,-1),(0,0),(2,-2), \\
(2,1),(4,-4),(4,3)\end{array}$ & - \\
\hline$\Pi_{3}(x)$ & $(0,-2),(0,-1),(0,0)$ & - & $(0,-2),(0,-1),(0,0)$ & - \\
\hline$\Pi_{4}(x)$ & $\begin{array}{l}(0,-3),(0,-2), \\
(0,-1),(0,0)\end{array}$ & - & $\begin{array}{l}(0,-3),(0,-2), \\
(0,-1),(0,0)\end{array}$ & - \\
\hline$\left(\begin{array}{l}x \\
2\end{array}\right)$ & {$[18]$} & {$[19]$} & {$[17]$} & $(0,-1),(0,2),(1,-1)$, \\
\hline$\left(\begin{array}{l}x \\
3\end{array}\right)$ & {$[35]$} & {$[35]$} & {$[35]$} & $\begin{array}{l}(0,0),(0,1),(0,2),(0,3), \\
(1,-1),(1,4),(3,-2),\end{array}$ \\
\hline$\left(\begin{array}{l}3,5),(6,-5),(6,8) \\
\left(\begin{array}{l}x \\
4\end{array}\right)\end{array}\right.$ & {$[34]$} & {$[1,-1),(1,4)$} & \\
\hline
\end{tabular}

TABLE 2

\section{Proofs}

We need some new concepts and also some lemmas for the proof of Theorem 3.1. A polynomial $f(x) \in \mathbb{C}[x]$ is called non-degenerate if it has at least three zeros of odd multiplicities.

Lemma 4.1 (Brindza [4]). Let $b$ be a non-zero rational number and $f(x) \in \mathbb{Q}[x]$ a non-degenerate polynomial. Then for the integral solutions $x, y$ of the hyperelliptic equation

$$
f(x)=b y^{2}
$$

we have $\max (|x|,|y|)<c_{1}$, where $c_{1}$ is an effectively computable constant depending only on $b$ and $f$.

Lemma 4.2 (Ping-Zhi [26]). Let $k$ be an integer with $k \geq 5, b$ an algebraic number and put $f_{k}(x)=\left(\begin{array}{l}x \\ k\end{array}\right)-b$. Then apart from the cases when $k=6, b=-\frac{10 \pm 7 \sqrt{7}}{1215}$, the polynomial $f_{k}(x)$ is non-degenerate. 
Further on, let $B_{k}(x)$ and $E_{k}(x)$ denote the $k$ th Bernoulli and Euler polynomial, respectively (see e.g. [28]).

Lemma 4.3 (Pintér and Rakaczki [27]). If $k$ is an integer with $k \geq 5$ and $a, b$ are complex numbers with $b \neq 0$ then the polynomial $\left(B_{k}(x)+\right.$ $a)^{2}+b$ is non-degenerate.

Lemma 4.4 (Rakaczki [29]). If $k$ is an integer with $k \geq 5$ and $a, b$ are complex numbers with $b \neq 0$ then the polynomial $\left(E_{k}(x)+a\right)^{2}+b$ is non-degenerate.

Now we have all the tools to prove Theorem 3.1.

Proof of Theorem 3.1. We split the proof into three parts according to the value of $k$. In each part we investigate the possible choices for $p(x)$ in turn.

The case $k \geq 5$. Assume first that $p(x)=\left(\begin{array}{l}x \\ k\end{array}\right)$. By (1) with $y=V_{n}$, we get

$$
y^{2}=D\left(\begin{array}{l}
x \\
k
\end{array}\right)^{2}+4 C(-B)^{n}
$$

for which by factoring the right-hand side we obtain

$$
y^{2}=D\left(\left(\begin{array}{l}
x \\
k
\end{array}\right)+2 \sqrt{\frac{-C(-B)^{n}}{D}}\right)\left(\left(\begin{array}{l}
x \\
k
\end{array}\right)-2 \sqrt{\frac{-C(-B)^{n}}{D}}\right) .
$$

Note that as $B= \pm 1$ and $C \neq 0$ the zeros of the two factors on the right-hand side of (4) must be distinct. Thus by Lemmas 4.1 and 4.2 it is enough to consider the cases where

$$
k=6, b=-\frac{10 \pm 7 \sqrt{7}}{1215} .
$$

Hence by a simple calculation we get that one of the factors of the righthand side of (4) is non-degenerate. Thus by Lemma 4.1 the theorem follows.

Now let $p(x)=\Pi_{k}(x)$. By (1) with $y=V_{n}$ we obtain that

$$
y^{2}=D \Pi_{k}(x)^{2}+4 C(-B)^{n} .
$$

Since $\Pi_{k}(x)=k !\left(\begin{array}{c}x+k-1 \\ k\end{array}\right)$, and a non-zero constant multiple of a nondegenerate polynomial is non-degenerate, by the previous argument the polynomial on the right-hand side of (5) is non-degenerate. Thus the theorem follows also in this case.

Assume that $p(x)=S_{k-1}(x)$. It is well-known that

$$
S_{k-1}(x)=\frac{1}{k}\left(B_{k}(x)-B_{k}(0)\right),
$$


where $B_{k}(x)$ is the $k$ th Bernoulli polynomial. Thus by (1) with $y=V_{n}$ we have

$$
y^{2}=\frac{D}{k^{2}}\left(\left(B_{k}(x)-B_{k}(0)\right)^{2}+\frac{4 C(-B)^{n} k^{2}}{D}\right) .
$$

Applying Lemma 4.3 with $a=-B_{k}(0)$ and $b=\frac{4 C(-B)^{n} k^{2}}{D} \neq 0$, the polynomial on the right-hand side of (6) is non-degenerate. So the theorem follows again by Lemma 4.1 .

Finally, let $p(x)=T_{k}(x)$. It is well-known that for all $k \in \mathbb{N}$

$$
T_{k}(x)=\frac{1}{2}\left(E_{k}(0)+(-1)^{x+1} E_{k}(x)\right)
$$

holds, where $E_{k}(x)$ is the $k$ th Euler polnomial. In the usual manner, (1) gives

$$
y^{2}=\frac{D}{4}\left(\left(E_{k}(x)+(-1)^{x+1} E_{k}(0)\right)^{2}+\frac{16 C(-B)^{n}}{D}\right) .
$$

Using Lemma 4.4 with $a=(-1)^{x+1} E_{k}(0)$ and $b=\frac{16 C(-B)^{n}}{D} \neq 0$, we obtain that the polynomial on the right-hand side of (7) is non-degenerate. Thus by Lemma 4.1 the theorem holds.

The case $k=4$. Take first $p(x)=\left(\begin{array}{l}x \\ 4\end{array}\right)$. Then (1) yields

$$
y^{2}=D\left(\begin{array}{l}
x \\
4
\end{array}\right)^{2}+4 C(-B)^{n}
$$

where $y=V_{n}$. If the discriminant of the polynomial on the righthand side is non-zero, then the polynomial is non-degenerate and the theorem is the consequence of Lemma 4.1. The discriminant of this polynomial is zero if and only if $4 C(-B)^{n}=\frac{-9 D}{16384}$, or $\frac{-D}{576}$, therefore we need to check only these two cases. In the first case we obtain the hyperelliptic equation

$$
\begin{aligned}
& y^{2}=D\left(\begin{array}{l}
x \\
4
\end{array}\right)^{2}-\frac{9 D}{16384}= \\
& =\frac{D}{147456}\left(4 x^{2}-12 x-1\right)\left(16 x^{4}-96 x^{3}+176 x^{2}-96 x+9\right)(2 x-3)^{2}
\end{aligned}
$$

and by Lemma 4.1 we are done. The second case gives the hyperelliptic equation

$$
y^{2}=D\left(\begin{array}{l}
x \\
4
\end{array}\right)^{2}-\frac{D}{576}=\frac{D}{576}\left(x^{4}-6 x^{3}+11 x^{2}-6 x-1\right)\left(x^{2}-3 x+1\right)^{2}
$$

and by Lemma 4.1 the theorem follows again. 
When $p(x) \in\left\{\Pi_{4}(x), S_{3}(x), T_{4}(x)\right\}$ the theorem can be verified by a similar argument. We omit the details.

The case $k \leq 3$. First note that when $p(x)=T_{3}(x)$ by a similar argument as in case of $k=4$ the theorem follows. Hence we may assume that either $k=2$, or $k=3, p(x) \in\left\{\left(\begin{array}{l}x \\ 3\end{array}\right), \Pi_{3}(x), S_{2}(x)\right\}$. Recall that in these cases we have $B=1$. We only consider an example, all the other possibilities can be handled similarly. Let $p(x)=\left(\begin{array}{l}x \\ 2\end{array}\right)$. Putting $y=V_{n}$ in (1) we get

$$
y^{2}=D\left(\begin{array}{l}
x \\
2
\end{array}\right)^{2}+4 C(-1)^{n}
$$

The discriminant of the polynomial on the right-hand side is zero if and only if $4 C(-1)^{n}=\frac{-D}{64}$. Thus this polynomial is non-degenerate, unless $256 \mid D$ is valid. However, as now $D=A^{2}+4$, a simple calculation gives that it is impossible. Therefore the right-hand side of (8) is nondegenerate and by Lemma 4.1 the theorem follows.

For the proof of Proposition 3.1 we need the following concept. Let $T_{k}(x)$ denote the Chebisev polynomial of degree $k$, i.e., $T_{0}(x)=2$, $T_{1}(x)=x$, and $T_{n+1}(x)=x T_{n}(x)-T_{n-1}(x)$ for $n \geq 1$. Further, we use the following result which is an immediate consequence of a theorem of Nemes and Pethő [21].

Lemma 4.5 (Nemes and Pethö [21]). Let $U_{n}$ be a non-degenerated binary recurrence sequence with $|B|=1$, and

$$
p(x)=a_{k} x^{k}+a_{k-1} x^{k-1}+\ldots+a_{0}
$$

be a polynomial with integer coefficients of degree $k \geq 2$. Let be $q=$ $-(-B)^{m} C / D$ and $E=2(k-1) a_{k-1}^{2}-4 k a_{k} a_{k-2}$. If (2) has infinitely many solutions $n, x$, then

$$
p(x)=\varepsilon \sqrt{q} T_{k}\left(\frac{2 k\left|a_{k}\right|}{\eta \sqrt{E}} x+\frac{2 a_{k-1}}{\eta \sqrt{E}}\right),
$$

where $\varepsilon$ and $\eta$ are either 1 or -1 .

Proof of Proposition 3.1. First we show that the recurrence sequences from Table 1 corresponding to the appropriate polynomials $p(x)$, can be constructed by the help of Lemma 4.5. We consider only one example, the others can be handled similarly. Let $p(x)=\left(\begin{array}{l}x \\ 2\end{array}\right)$. Then $p(x)$ can be written in the form

$$
p(x)=\frac{1}{16} T_{2}(2 \sqrt{2} x-\sqrt{2})=\frac{1}{16}\left(8 x^{2}-8 x\right) .
$$

From this by Lemma 4.5, it follows that if equation (2) has infinitely many solutions then the parameters of the binary recurrence sequence 
$U$ must satisfy $\frac{C}{D}=-\frac{1}{256}$. Choosing $U_{0}=1, U_{1}=253, A=254$ with $B=-1$ we get that $\frac{C}{D}=\frac{-252}{64512}=-\frac{1}{256}$. Hence we conclude that the binary recurrence sequence belonging to the parameters of the first row of Table 1 and the polynomial $p(x)=\left(\begin{array}{l}x \\ 2\end{array}\right)$ satisfy the conditions of Lemma 4.5.

Now we prove that with these choices of the parameters, equation (2) actually has infinitely many solutions. Since the companion polynomial of $U$ is $x^{2}-254 x+1$, we have

$$
U_{n}=\frac{3 \sqrt{7}+8}{16}(127+48 \sqrt{7})^{n}-\frac{3 \sqrt{7}-8}{16}(127-48 \sqrt{7})^{n} \quad(n=0,1, \ldots) .
$$

Let $W=\left\{W_{n}\right\}_{n=0}^{\infty}$ be the ternary recurrence sequence defined by the initial values $W_{0}=2, W_{1}=23, W_{2}=359$ and by the recurrence relation $W_{n}=17 W_{n-1}-17 W_{n-2}+W_{n-3}(n \geq 3)$. Then the companion polynomial of $W$ is

$$
x^{3}-17 x^{2}+17 x-1=\left(x^{2}-16 x+1\right)(x-1) .
$$

Hence we have

$$
W_{n}=\frac{3+\sqrt{7}}{4}(8+3 \sqrt{7})^{n}+\frac{3-\sqrt{7}}{4}(8-3 \sqrt{7})^{n}+\frac{1}{2} 1^{n} .
$$

Since $U_{n}$ can be written as $U_{n}=\frac{1}{16}\left((8+3 \sqrt{7})^{2 n+1}+(8-3 \sqrt{7})^{2 n+1}\right)$, it can be easily verified that for all $n=0,1, \ldots$ we have $U_{n}=\left(\begin{array}{c}W_{n} \\ 2\end{array}\right)$. Thus with the choice $x=W_{n}$ equation (2) has infinitely many solutions in $n, x$.

Proof of Theorem 3.2. As we explained before formulating the theorem, to prove the statement it is sufficient to consider equation (3) with our special settings, i.e.

$$
Y^{2}=D\left(a^{2} X^{4}+2 a c X^{3}+\left(c^{2}+2 a d\right) X^{2}+2 c d X+d^{2}\right)+4 C(-B)^{n} e^{2} .
$$

The discriminant of the polynomial on the right-hand side is

$$
\Delta=256 D^{3} a^{4} C^{2} e^{4}\left(16 a^{2} D d^{2}+64 a^{2} C(-B)^{n} e^{2}-8 a D c^{2} d+c^{4} D\right) .
$$

Since by our conditions $D a e \neq 0$, and $U$ is non-degenerate therefore $C \neq 0$, thus if

$$
8 a D d\left(2 a d-c^{2}\right) \neq-64 a^{2} C(-B)^{n} e^{2}-c^{4} D,
$$

then $\Delta \neq 0$. Hence, by Lemma 4.1 the solutions $n, x$ of (2) can be effectively determined.

The solutions can be determined explicitly in the following way. In what follows, we use certain procedures of the program package Magma and also Magma programs of Bruin and Stoll [5] and Tengely [36]. We emphasize that all the procedures we use or mention are known from 
the literature. The novelty at this point is only that we put them together in order to get a complete algorithm.

First, by the command HyperellipticCurve we define the hyperelliptic curve

$$
Y^{2}=h(X),
$$

where $h(X):=h_{4} X^{4}+h_{3} X^{3}+h_{2} X^{2}+h_{1} X+h_{0}$ is the right-hand side of (3). If $h_{4}$ is a perfect square then one can use Runge's method to solve (9). In fact, by the help of a Magma program of Tengely, all solutions can be determined in this case (see [36]). Otherwise, we try to determine some rational points on the curve (9) with the help of the procedure Points. If we cannot get any rational points, then most probably (9) has no rational solutions at all. This can be very efficiently checked by the procedure TwoCoverDescent of Bruin and Stoll. (For the description of this procedure and some examples see [5].)

Assume now that with the procedure Points we obtained some rational points on (9). Suppose that there is a point $\left(X_{0}, Y_{0}\right)$ among them such that $Y_{0}=0$. Let $\left(X_{0}, 0\right)=\left(\frac{x_{1}}{x_{2}}, 0\right)$ be such a point of the curve. Then using the substitutions $U=x_{2} X-x_{1}$ and $V=x_{2}^{2} Y$, noting that $h\left(X_{0}\right)=0$, we obtain an equation of the shape

$$
V^{2}=t_{4} U^{4}+t_{3} U^{3}+t_{2} U^{2}+t_{1} U
$$

with some $t_{i} \in \mathbb{Z}(i=1, \ldots, 4)$. Factorizing the right-hand side we get that

$$
s V_{1}^{2}=U
$$

and

$$
s V_{2}^{2}=t_{4} U^{3}+t_{3} U^{2}+t_{2} U+t_{1}
$$

with some integers $s, V_{1}, V_{2}$. Equation (10) implies that $s \mid U$, hence (11) yields that $s \mid t_{1}$. Thus to solve our original problem it is sufficient to find the integral points on finitely many elliptic curves given by (11). This can be done with the procedure IntegralPoints. Following the substitutions backwards we obtain all integer solutions of (9).

Finally, consider the case when the procedure Points finds only rational points on (9) with nonzero second coordinates. In this case by the help of certain birational transformations (9) can be transformed into an elliptic curve. For the theory of birational transformations see e.g. Harada and Lang [14], Connell [11], Tzanakis [38], Hermann [15] and the references given there. To resolve (9) completely, one can use the procedure IntegralQuarticPoints of Magma (which is 
actually based upon results from [38]). One needs to call the procedure IntegralQuarticPoints by (9) and one of the above mentioned points. In this way we can get all integral solutions of (9) also in this case.

We implemented our algorithm for the resolution of (9) in Magma. The program can be downloaded from www.math.klte.hu/ tkovacs or can be requested from the author.

From the solutions of equation (3) all solutions $n, x$ of the original equation (2) can be easily determined.

Proof of Theorem 3.3. We split the proof into three parts. We start with equations which turn to be unsolvable locally. Then we deal with equations which can be reduced to elliptic equations. Finally, we prove the theorem for those equations which can be reduced to genus 1 equations. In all cases we give the proof only for one equation as the other ones can be handled similarly.

Throughout the proof we shall use the well-known facts that $L$ is the associate sequence of $F$ and $Q$ is the associate sequence of $P$.

Locally unsolvable equations. In this part of the proof we deal with those equations which turn out to be locally unsolvable for some prime. The following equations belong to this group: $Q_{n}=\Pi_{2}(x), \Pi_{4}(x)$.

As an example, take the equation $Q_{n}=\Pi_{2}(x)$. Writing $y=P_{n}$ in (1), we get the equations

$$
y^{2}=8(x(x+1))^{2} \mp 8 .
$$

A simple calculation modulo 16 leads to a contradiction. Hence equation $Q_{n}=\Pi_{2}(x)$ does not have any integer solutions. We note that our algorithm described in the proof of Theorem 3.2 provides the same conclusion.

Elliptic equations. In this part we handle those equations which can be reduced to elliptic equations. The following equations belong to this set: $Q_{n}=S_{2}(x),\left(\begin{array}{l}x \\ 3\end{array}\right)$ and $U_{n}=\Pi_{3}(x)$, with $U_{n} \in\left\{F_{n}, L_{n}, P_{n}, Q_{n}\right\}$.

As an example, consider the equation $P_{n}=\Pi_{3}(x)$. With the substitution $y=Q_{n}$, (1) yields

$$
y^{2}=8(x(x+1)(x+2))^{2} \pm 4 .
$$

With the substitution $x_{1}=2(x+1)^{2}$ the right-hand side can be transformed to a polynomial of degree 3 , therefore we obtain the elliptic equations

$$
y^{2}=x_{1}^{3}-4 x_{1}^{2}+4 x_{1} \pm 4
$$


With the procedure IntegralPoints of Magma one can compute the integer points of these curves, and then determine the solutions $n, x$ of (2). The solutions are exactly the ones listed in Table 2 .

Genus 1 equations. In this part we consider those equations which can be reduced to genus 1 equations. All the equations considered which are not mentioned so far belong to this group.

Consider the equation $F_{n}=\Pi_{4}(x)$. With $y=L_{n}$ and $x_{1}=x^{2}+3 x$ by (1) we get the equation

$$
y^{2}=5 x_{1}^{4}+20 x_{1}^{3}+20 x_{1}^{2}+4(-1)^{n} .
$$

If $n$ is even then directly, if $n$ is odd then after the substitution $x_{2}=$ $x_{1}+1$ we can apply the procedure IntegralQuarticPoints of Magma to compute the integer solutions of this equation. Then we easily get the solutions $n, x$ of the original equation (2). The solutions are exactly the ones listed in Table 2.

\section{ACKnowledgement}

The author is grateful to Lajos Hajdu, Ákos Pintér and Szabolcs Tengely for their useful remarks and help. The author thanks Attila Pethö for his advice and helpful suggestions on an earlier version of the paper.

\section{REFERENCES}

[1] B. U. Alfred, On square Lucas numbers, Fib. Quarterly 2 (1964), 11-12.

[2] A. Baker, The Diophantine equation $y^{2}=a x^{3}+b x^{2}+c x+d$, J. London Math. Soc. 43 (1968), 1-9.

[3] W. Bosma, J. Cannon, C. Playoust, The Magma algebra system. I. The user language., J. Symbolic Comput. 24 (1997), 235-265.

[4] B. Brindza, On S-integral solutions of the equation $y^{m}=f(x)$, Acta. Math. Hungar. 44 (1984), 133-139.

[5] N. Bruin, M. Stoll Two-cover descent on hyperelliptic curves (to appear).

[6] Y. Bugeaud, M. Mignotte, S. Siksek, Classical and modular approaches to exponential Diophantine equations. I. Fibonacci and Lucas perfect powers., Ann. of Math. (2) 163 (2006), 969-1018.

[7] J. H. E. Cohn, Square Fibonacci numbers, Etc., Fib. Quarterly 2 (1964), $109-113$.

[8] J. H. E. Cohn, On square Fibonacci numbers, J. London Math. Soc. 39 (1964), $537-540$.

[9] J. H. E. Cohn, Lucas and Fibonacci numbers and some Diophantine equations, Proc. Glasgow Math. Assoc. 7 (1965), 24-28.

[10] J. H. E. Cohn, Perfect Pell powers, Glasgow Math. J. 38 (1996), 19-20.

[11] I. Connell, Addendum to a paper of Harada and Lang, J. Algebra 145 (1992), 463-467.

[12] J. Gebel, A. Pethő, H. G. Zimmer, Computing integral points on elliptic curves, Acta Arith. 68 (1994), 171-192. 
[13] L. Hajdu, Á. Pintér, Combinatorial diophantine equations, Publ. Math. Debrecen 56 (2000), 391- 403.

[14] K. Harada, M. L. Lang, Some elliptic curves arising from the Leech lattice, J. Algebra 125 (1989), 298-310.

[15] E. Herrmann, Bestimmung aller ganzzahligen Lösungen quartischer elliptischer diophantischer Gleichungen unter Verwendung von Linearformen in elliptischen Logarithmen, Section 1.6. (diploma thesis, 1998).

[16] T. Kovács, Combinatorial Diophantine equations - the genus 1 case, Publ. Math. Debrecen 72 (2008), 243-255.

[17] W. L. McDaniel, Triangular numbers in the Pell sequence, Fib. Quarterly 34 (1996), 105-107.

[18] L. Ming, On triangular Fibonacci numbers, Fib. Quarterly 27 (1989), 98-108.

[19] L. Ming, On triangular Lucas numbers, Applications of Fibonacci numbers 4 (1991), 231-240.

[20] L. Moser, L. Carlitz, Problem H-2, Fib. Quarterly 1 (1963), 46.

[21] I. Nemes, A. Pethő, Polynomial values in linear recurrences II, J. Number Theory 24 (1986), 47-53.

[22] C. S. Ogilvy, Tomorrow's math, unsolved problems for the amateur, Oxford Univ. Press (1962), p.100.

[23] A. Pethö, On the Solution of the Equation $G_{n}=P(x)$, in Fibonacci Numbers and Their Applications, D. Reidel Publ. Comp., 1986, 193-201.

[24] A. Pethő, Diofantoszi egyenletek effektiv és explicit megoldása (in Hungarian), Academic doctoral dissertation, Hungarian Academy of Sciences, 1990, 114 pp.

[25] A. Pethő, The Pell sequence contains only trivial perfect powers, Colloq. Math. Soc. János Bolyai 60 Sets, Graphs and Numbers Budapest (Hungary), 1991, 561-568.

[26] Y. Ping-Zhi, On a special diophantine equation $a\left(\begin{array}{l}x \\ n\end{array}\right)=b y^{r}+c$, Publ. Math. Debrecen, 44 (1994), 137-143.

[27] Á. Pintér, Cs. Rakaczki, On the zeros of shifted Bernoulli polynomials, Appl. Math. Comput. 187 (2007), 379-383.

[28] H. Rademacher, Topics in analytic number theory, Die Grundlehren der math. Wissenschaften, Band 169, Springer-Verlag, Berlin, 1973, ix+320 pp.

[29] Cs. Rakaczki, On some Diophantine results related to Euler polynomials, Periodica Math. Hungar. (to appear).

[30] A. P. Rollett, Problem 5080, Amer. Math. Monthly, 70 (1963), 216.

[31] T. N. Shorey, R. Tijdeman, Exponential diophantine equation, Cambridge University Press, 1986, pp.X+280

[32] R. J. Stroeker, N. Tzanakis, Solving elliptic diophantine equations by estimating linear forms in elliptic logarithms, Acta Arith. 67 (1994), 177-196.

[33] R. J. Stroeker, N. Tzanakis, Computing all integer solutions of a genus 1 equation, Comp. Math. 72 (2003), 1935-1946.

[34] L. Szalay, Some polynomial values in binary recurrences, Revista Colombiana de Matemáticas 35 (2001), 99-106.

[35] L. Szalay, On the resolution of the equation $U_{n}=\left(\begin{array}{l}x \\ 3\end{array}\right)$ and $V_{n}=\left(\begin{array}{l}x \\ 3\end{array}\right)$, Fib. Quarterly 40 (2002), 9-12.

[36] Sz. Tengely, On the Diophantine equation $F(x)=G(y)$, Acta Arith. 110 (2003), 185-200. 
[37] Sz. Tengely, Finding g-gonal numbers in recurrence sequences (to appear).

[38] N. Tzanakis, Solving Elliptic Diophantine Equations by estimating Linear Forms in Elliptic Logarithms. The case of Quartic Equations, Acta Arith. $\mathbf{7 5}$ (1996), 165-190.

[39] O. Wyler, In the Fibonacci series $F_{1}=1, F_{2}=1, F_{n+1}=F_{n}+F_{n-1}$ the first, second and twelfth terms are squares, Amer. Math. Monthly 71 (1964), $221-222$.

T. KOVÁCS

University of Debrecen, Institute of Mathematics

DEBRECEN

P.O. Box 12 .

$\mathrm{H}-4010$

HUNGARY

E-mail address: tkovacs@math.klte.hu 